\title{
Power Curve Characterization II: modelling using polynomial regression
}

\author{
A. Llombart ${ }^{1}$, S. J. Watson ${ }^{2}$, J.M. Fandos ${ }^{1}$ and D. Llombart ${ }^{1}$ \\ ${ }^{1}$ Department of Electrical Engineering \\ Fundacion CIRCE, Zaragoza University \\ C/ Maria de Luna 3, 50018 Zaragoza (Spain) \\ Phone/Fax number:+34 976 762398, e-mail: llombart@unizar.es \\ ${ }^{2}$ Centre for Renewable Energy Systems Technology, Loughborough University, Ashby Road, Loughborough, \\ Leicestershire, LE11 3TU, UK \\ Phone: +44 1509 222421, Fax: +44 1509 610031, e-mail: s.j.watson@lboro.ac.uk
}

\begin{abstract}
Accurate determination of wind turbine performance is necessary for economic operation of a wind farm. We propose modifications to the basic polynomial method to take account of distance between the meteorological mast and the wind turbine and also the complexity of the terrain. The methods are evaluated using data from operational wind farms and the methods are compared to a modified IEC 61400-12 bin method.
\end{abstract}

\section{Key words}

Wind energy, power curve, bin method, power performance, and polynomial regression

\section{Introduction}

The cost effective operation and maintenance of wind farms requires accurate and informed condition monitoring of the wind turbines. One such technique is to analyse over time the power performance of the turbines.

For the latest wind turbines, e.g. $>600 \mathrm{~kW}$, there is limited data available to assess the long-term power performance of these machines. In addition, in Spain there are concerns in relation to the long-term operation in sites with highly complex terrain and high levels of turbulence intensity [1] - [4].

To assess the efficiency of a wind turbine the relationship between the wind mean speed taken at the meteorological mast and the power generated by each wind turbine must be found. But the best method to do this is not clear. There are some factors that complicate this relationship:

1. The time delay in the wind speed propagation and how this affects the correlation between mast and turbine wind speed;

2. The topography of the wind farm site;

3. The presence of nearby obstacles.

Moreover, the increasing size of wind farms makes the effect of these factors worse due to the increasing distance between some of the wind turbines and the meteorological mast.
Several studies have been carried out on this topic. In [1] a Neural Network (NN) with Kalman filter training is described. This work is improved in [2] where the authors achieved good results using NN. However, to achieve these results, they used data from two meteorological towers, and the furthest turbine was only $600 \mathrm{~m}$ from the nearest tower mast.

In [3], a method is presented that allows the detection of a decrease in the efficiency of any turbine up to a distance of $4 \mathrm{~km}$ from the meteorological mast. This method does not provide a power estimation, however, so it cannot be used for anything more than efficiency evaluation. In addition, this method takes six weeks to detect any problem with the turbine power performance.

Finally, in [4] it is suggested that the use of NNs is suited to the problem of power performance evaluation but the issue of distance from mast to turbine is not really addressed.

References [1], [2] and [4] are based solely on the use of Neural Networks. These references compare the use of NNs with relatively simple stochastic methods and conclude that the NN approach is superior, however, the stochastic methods chosen are overly simplistic.

In this paper, different stochastic methods that can be used to solve the problem are reviewed. Here we concentrate on the polynomial regression method. The method has been applied using the wind speed at the meteorological tower and the wind speed at the nacelle of each turbine, this has allowed to compare both approaches. Moreover, some other modifications has been proposed in order to improve the results achieved with the polynomial regression method.

To show the suitability of this method the results are tested with real data from a wind farm situated in Aragon, Spain. In addition, the polynomial method is compared to the modified bin method proposed in [3]. 


\section{Stochastic methods}

The first stochastic method that has been used to infer the power of any wind turbine from the speed at the meteorological mast is to derive it from the manufacturer's power curve, possibly taking into account the correction due to the changes in air density. This method can also use the wind speed measured at the nacelle of each turbine.

The power curve given by the manufacturer has been obtained in ideal conditions, and clearly the turbines in an operational wind farm are not placed ideally in relation to the meteorological mast in the wind farm site. This means that the 'ideal' power curve is not always suitable for assessing the power performance of a wind turbine operating in a wind farm, particularly if the terrain is relatively complex.

To get a more accurate definition of the power curve for each turbine, this can be calculated from real historical data from the meteorological mast and the different turbines in the wind farm. There are some probabilistic methods to obtain the relationship between the mast and turbine:

- Bin method

- Regression

In this paper, we are going to consider the regression method. The relationship between the variables is adjusted using a polynomial. The data is first normalised, as stated in the IEC 61400-12 standard, to a density reference value.

The power curve of a wind turbine has three clearly defined parts. Firstly, between $0 \mathrm{~m} / \mathrm{s}$ and the cut-in wind speed the production is zero, then the power output increases following a cubic relationship with wind speed until the rated wind speed, and finally, depending on the wind turbine model, the power output is regulated to stay almost constant until the cut-out or furling wind speed. This three-part shape suggests that different polynomial relationships should be used for different wind speed ranges.

There will inevitably be certain wind directions in which obstacles, in particular other turbines, will affect either the production of a wind turbine or the wind speed measurement at the meteorological tower mast.

To take account of these phenomena some modifications in the polynomial regression method have been proposed in order to increase the accuracy of the characterization of the power performance of the wind turbines. These modifications have been tested. The different modifications proposed are explained in the following paragraph.

\section{Methodology}

To achieve a good characterisation of the relationship between power output at a wind turbine and the wind speed either at a meteorological mast or at the nacelle of each turbine, we have used historical data from an operational wind farm. Due to confidentiality issues it is not allowed to present it in detail but, in order to understand the type of the terrain and to present the situation of the wind turbines whose data are used to undertake the study, a simplified map is presented (Fig. 1).

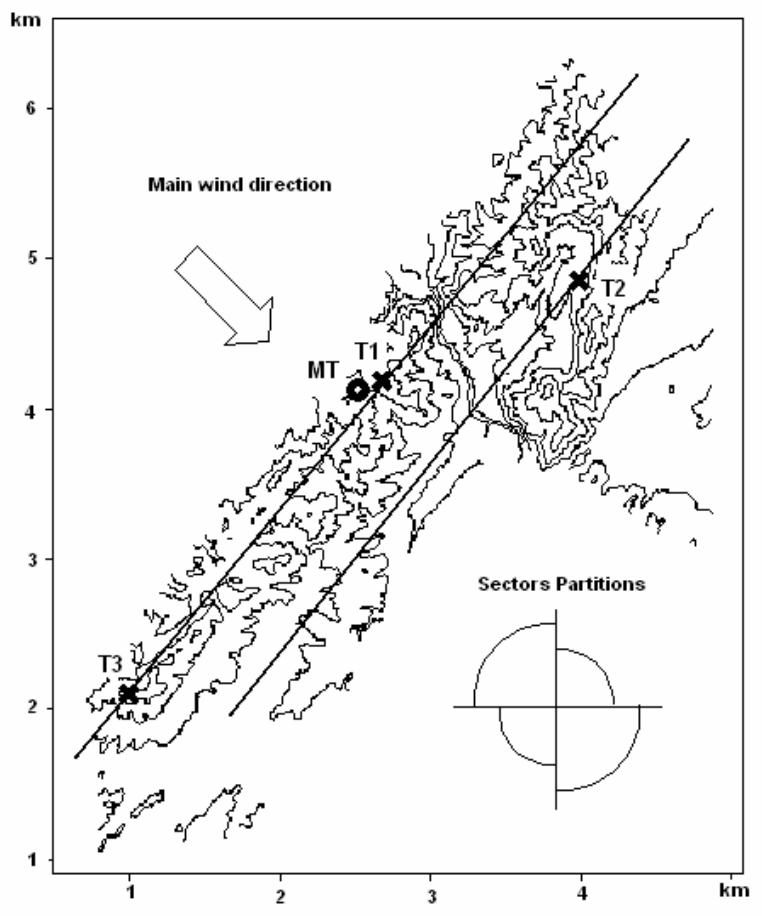

Figure 1.- A simplified view of the wind farm

In this map, it can be seen that the terrain is extremely complex, the turbines are disposed in two lines (following, more or less, the straight lines represented in the map) and the main wind direction is indicated by the big arrow in the top left corner.

The turbines considered, have been chosen taking into account their distance to the meteorological tower, turbine 1 is near of it, and turbine 2 and 3 are further. The objective of this election is to corroborate the influence of the distance in the characterization method. The main direction of the wind is also shown.

We have considered a number of approaches to improve the polynomial method, those approaches are described below. Validation of the different methods described is carried out by comparing the predicting wind turbine power outputs with the actual power outputs and by calculating the mean squared error.

\section{A. All data}

Initially, the data were analysed as a group and the relationship between mast and turbine determined. This 
was used to test the influence of distance and as a base case against which to compare the different methods described below.

The approach proposed limits the polynomial calculated to the fifth power of the independent variable and, due to the limitation of the tool utilized (Excell by Microsoft), all the different terms of the polynomial have been taken into consideration, that is, the different expressions obtained are as that showed below:

$$
P=a_{0}+a_{1} V+\cdots+a_{5} V^{5}
$$

\section{B. Partitioning the power curve by wind speed}

For an ideal power curve, the portioning of the curve for the purposes of fitting a polynomial is fairly clear. However, if a turbine is a significant distance from the turbine, the correlation between the wind speeds at mast and turbine reduce and the effective power curve observed may not be so clearly partitioned and may differ from turbine to turbine depending on the distance.

As a first step, the partitions for each turbine used in the study have been chosen identically for all the cases. This has allowed comparing the results easily.

In the next figure it can be seen the limits proposed, these have been chosen using practical methods (some try and error probes arround the theoretical ranges).

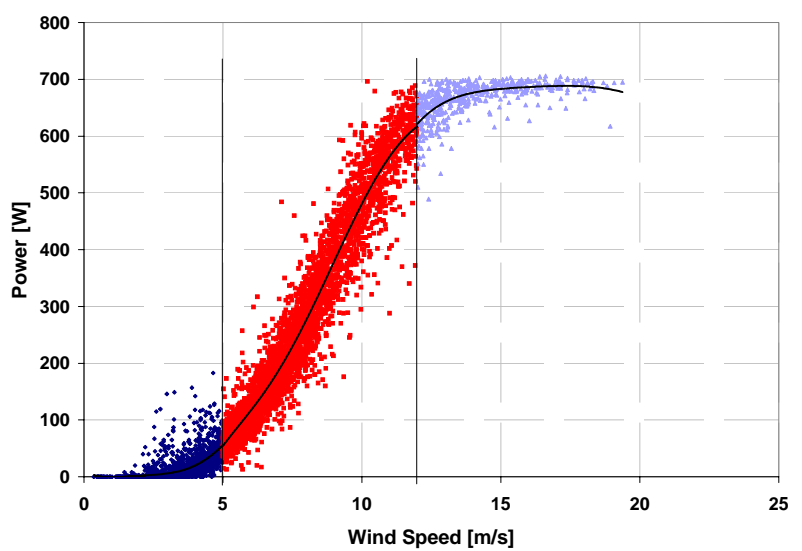

Figure 2.-P-V curve for wind turbine 1 and wind speed partition

\section{Fitting different polynomials by wind direction}

If the wind farm site is complex, the correlation between mast and turbine wind speed will be different for different direction sectors. This suggests that the power curve should be fitted using a different polynomial curve for different direction sectors.

\section{Partitioning by both wind speed and direction}

Clearly, partitioning of the power curve can be made for both wind speed and these partitions can be changed for different direction sectors before polynomials are fitted.
These methods are compared with the bin method described in [3]. It must be noted that using the mean squared error to compare the different methods is a valid approach.

\section{Results}

As it is explained in [3] the first step before any analysis process is to verify the quality of the data that are going to be used.

The different data used for this study have been filtered previously to avoid the consideration of any wrong data, and a set of right data have been obtained for each turbine and the meteorological tower.

The different methods explained in the paragraph 3 have been applied to the three turbines using both the wind speed taken at the meteorological mast and the wind speed taken in the nacelle of each turbine. These results can be found in paragraph A. The comparison between these results and those obtained in [3] is analyzed in paragraph B.

\section{A. Experimental results for the polynomial regression based methods}

The following tables show the results obtained in the different approaches. There is a table for each wind turbine and for each wind speed used; in these tables the results of each method are shown. The mean squared error is presented and for the different modifications also is presented the improvement measure as a percentage of the first method.

Firstly, the tables corresponding to the use of the wind speed at the meteorological mast are presented.

TABLE I. - Characterization results for turbine 1 using the wind speed at the MT

\begin{tabular}{|l|c|c|}
\hline Turbine 1 & MSE & Improv. (\%) \\
\hline Method A & 1383,26 & \\
\hline Method B & 1358,41 & $1,80 \%$ \\
\hline Method C & 1247,05 & $9,85 \%$ \\
\hline Method D & 1215,91 & $12,10 \%$ \\
\hline
\end{tabular}

TABLE II. - Characterization results for turbine 2 using the wind speed at the MT

\begin{tabular}{|l|c|c|}
\hline Turbine 2 & MSE & Improv. (\%) \\
\hline Method A & 2773,11 & \\
\hline Method B & 2732,31 & $1,47 \%$ \\
\hline Method C & 1882,83 & $32,10 \%$ \\
\hline Method D & 1851,17 & $33,25 \%$ \\
\hline
\end{tabular}


TABLE III. - Characterization results for turbine 3 using the wind speed at the MT

\begin{tabular}{|l|c|c|}
\hline Turbine 3 & MSE & Improv. (\%) \\
\hline Method A & 5539,53 & \\
\hline Method B & 5502,88 & $0,66 \%$ \\
\hline Method C & 4653,09 & $16,00 \%$ \\
\hline Method D & 4592,86 & $17,09 \%$ \\
\hline
\end{tabular}

Finally, the tables corresponding to the use of the wind speed at the nacelle of each wind turbine are presented. It must be remembered that when the data of wind direction are required they must be taken from the meteorological mast.

TABLE IV. - Characterization results for turbine 1 using the wind speed at the nacelle

\begin{tabular}{|l|c|c|}
\hline Turbine 1 & MSE & Improv. (\%) \\
\hline Method A & 118,19 & \\
\hline Method B & 81,26 & $31,25 \%$ \\
\hline Method C & 108,51 & $8,20 \%$ \\
\hline Method D & 78,11 & $33,92 \%$ \\
\hline
\end{tabular}

TABLE V. - Characterization results for turbine 2 using the wind speed at the nacelle

\begin{tabular}{|l|c|c|}
\hline Turbine 2 & MSE & Improv. (\%) \\
\hline Method A & 164,04 & \\
\hline Method B & 118,53 & $27,75 \%$ \\
\hline Method C & 127,23 & $22,44 \%$ \\
\hline Method D & 94,25 & $42,55 \%$ \\
\hline
\end{tabular}

TABLE VI. - Characterization results for turbine 3 using the wind speed at the nacelle

\begin{tabular}{|l|c|c|}
\hline Turbine 3 & MSE & Improv. (\%) \\
\hline Method A & 174,44 & \\
\hline Method B & 137,28 & $21,31 \%$ \\
\hline Method C & 157,39 & $9,77 \%$ \\
\hline Method D & 128,50 & $26,34 \%$ \\
\hline
\end{tabular}

It can be said that, for this wind farm, the wind speed taken at the nacelle is more suitable to characterize the power performance of the wind turbine.

The improvements achieved taking in account the modifications are bigger considering the wind speed that considering the wind direction. This phenomenon is opposite to that observed using the wind speed at the meteorological mast. The results are completely logical, while in the first case the distance between the MT and the turbine is important and makes that the different directions considered affect the relationship between the power and the wind speed, in this last case, the wind speed taken by the anemometer at the nacelle it is almost not influenced by the wind direction.
The improvements allowed considering the partition in wind speed are almost the same in all the cases studied (if the absolute values are compared).

B. Comparison between the polynomial regression based methods and the bin method ones

Looking at the results got in [3] it can be said that the two methods are similar for the task of representing the performance of the power in wind turbines.

In relation with the different modifications proposed, the consideration of different sector always improve the results obtained, and for the method based in polynomial regression the modification based on the wind speed partition is important in the case that the wind speed at the nacelle is considered.

\section{Conclusion}

The usefulness of polynomial regression in power performance assessment of wind turbines is demonstrated. Modifications to the basic polynomial method have been proposed which take account of distance and complexity of terrain. The value of these methods has been determined using actual wind farm data.

\section{Acknowledgement}

This work has been carried out with funding from the Spanish Ministerio de Ciencia y Tecnologia (projects: DPI2003-09731 and FIT-120000-2004-182) and the CaiEuropa Program.

\section{References}

[1] S. Li, D. C. Wunsch, E. A. O’Hair and M. G. Giesselmann, "Wind turbine power estimation by neural networks with kalman filter training on a SIMD parallel machine”, International Joint Conference on Neural Networks, Vol. 5, pp 3430 3434, July 1999

[2] S. Li, D. C. Wunsch, E. A. O’Hair and M. G. Giesselmann, "Using Neural Networks to Estimate Wind Turbine Power Generation”, IEEE trans. On Energy Conversion, Vol. 16, $\mathrm{N}^{\mathrm{o}} 3$, pp 276-282, September 2001

[3] A. Llombart, S. J. Watson, D. Llombart, and J. M. Fandos, "Power Curve Characterisation I: improving the bin method"; ICREPQ'05

[4] S. Kélouwani, K. Agbossou, "Nonlinear Model Identification of Wind Turbine with a Neural Network”, IEEE trans. On Energy Conversion, Vol. 19, № 3, pp 607-612, September 2004 